\title{
Effects of Liposome and Cardiolipin on Folding and Function of Mitochondrial Erv1
}

\author{
Xiaofan Tang ${ }^{1,2,3}\left(\mathbb{D}\right.$, Lynda K Harris $4,5,6 \mathbb{D}$ and Hui Lu ${ }^{1, *(\mathbb{D})}$ \\ 1 School of Biological Sciences, Faculty of Biology, Medicine and Health, The University of Manchester, \\ Manchester M13 9PT, UK; xf.tang@siat.ac.cn \\ 2 Department of Materials, Faculty of Science and Engineering, The University of Manchester, \\ Manchester M13 9PT, UK \\ 3 Shenzhen Engineering Laboratory of Nanomedicine and Nanoformulations, Shenzhen Institutes of \\ Advanced Technology (SIAT), Chinese Academy of Sciences, Shenzhen 518055, China \\ 4 Division of Pharmacy and Optometry, School of Health Sciences, Faculty of Biology, Medicine and Health, \\ The University of Manchester, Manchester M13 9PL, UK; Lynda.K.Harris@manchester.ac.uk \\ 5 Maternal and Fetal Health Research Centre, Faculty of Biology, Medicine and Health, The University of Manchester, \\ Manchester M13 9WL, UK \\ 6 Maternal and Fetal Health Research Centre, Manchester University NHS Foundation Trust, \\ Manchester Academic Health Sciences Centre, St Mary's Hospital, Manchester M13 9WL, UK \\ * Correspondence: hui.lu@manchester.ac.uk; Tel.: +44-161-2751553; Fax: +44-161-3065201
}

Received: 12 November 2020; Accepted: 8 December 2020; Published: 10 December 2020

\begin{abstract}
Erv1 (EC number 1.8.3.2) is an essential mitochondrial enzyme catalyzing protein import and oxidative folding in the mitochondrial intermembrane space. Erv1 has both oxidase and cytochrome $c$ reductase activities. While both Erv1 and cytochrome $c$ were reported to be membrane associated in mitochondria, it is unknown how the mitochondrial membrane environment may affect the function of Erv1. Here, in this study, we used liposomes to mimic the mitochondrial membrane and investigated the effect of liposomes and cardiolipin on the folding and function of yeast Erv1. Enzyme kinetics of both the oxidase and cytochrome $c$ reductase activity of Erv1 were studied using oxygen consumption analysis and spectroscopic methods. Our results showed that the presence of liposomes has mild impacts on Erv1 oxidase activity, but significantly inhibited the catalytic efficiency of Erv1 cytochrome $c$ reductase activity in a cardiolipin-dependent manner. Taken together, the results of this study provide important insights into the function of Erv1 in the mitochondria, suggesting that molecular oxygen is a better substrate than cytochrome $c$ for Erv1 in the yeast mitochondria.
\end{abstract}

Keywords: sulfhydryl oxidase; MIA pathway; mitochondrial membrane; enzyme kinetics

\section{Introduction}

Mitochondria are important organelles in eukaryotic cells, which produce most of the energy for biological processes. Since about $99 \%$ of proteins in mitochondria are imported from the cytosol, mitochondria have dedicated protein import systems for mitochondrial protein biogenesis [1]. Protein import and oxidative folding, conjugated with disulphide bond formation, is a unique protein import mechanism in the mitochondria intermembrane space (IMS), which is executed by the mitochondrial import and assembly (MIA) pathway [2-5]. Erv1 (essential for respiration and viability) [1] in yeast, or called ALR (augmenter of liver regeneration) in mammals, is an essential component of the MIA pathway, which works together with Mia40 to catalyze the oxidative folding of the newly imported substrate proteins in the IMS [6-8]. Mia40 acts as an oxidoreductase, interacting with the substrate proteins and transferring disulphide bonds to the substrates. Erv1, a FAD-binding protein, serves as a disulphide bond generator to reoxidize the reduced Mia40. Then, the reduced Erv1 is regenerated 
by passing electrons to molecular oxygen (acting as an oxidase) or cytochrome $c$ (as cytochrome $c$ reductase) via the flavin cofactor (Figure 1A) [9-11].
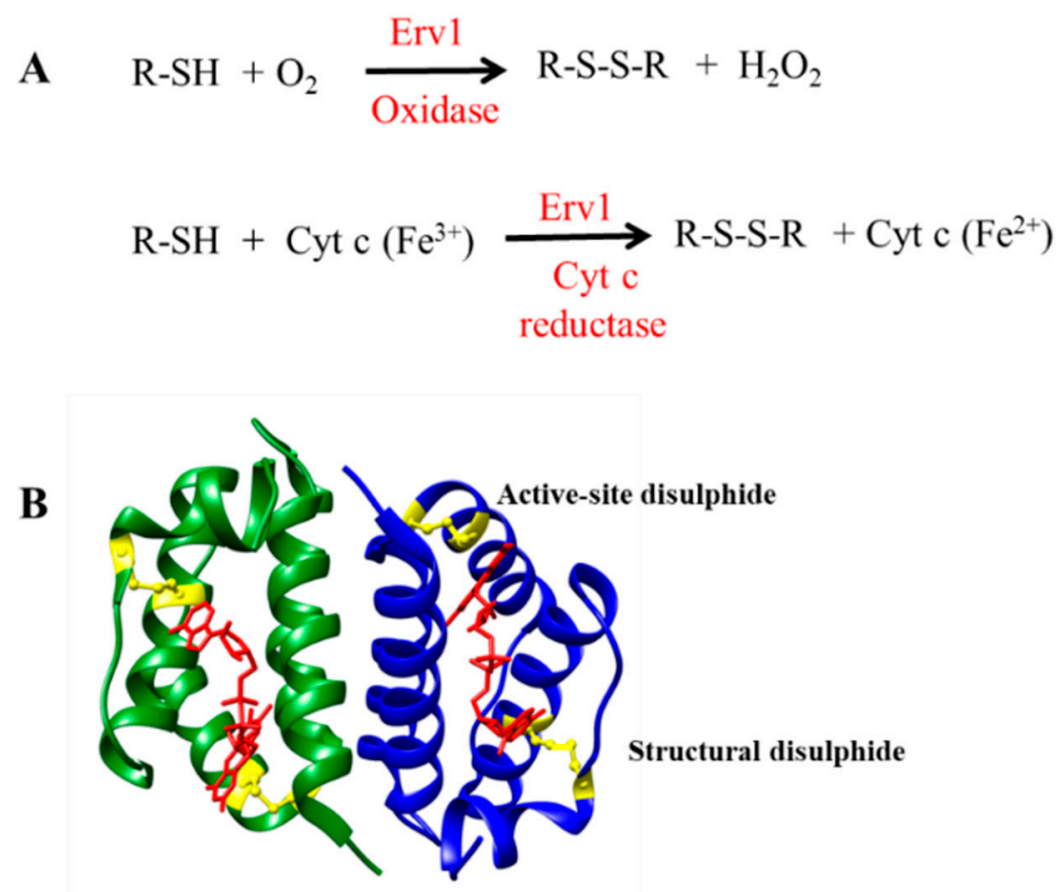

Figure 1. Function and structure of Erv1. (A) The reactions catalyzed by Erv1 as an oxidase and a cytochrome $c$ reductase, respectively. (B) X-ray structure of the C-terminal domain of Erv1. The active site disulphide and the structural disulphide are shown in yellow and FAD is shown in red.

Erv1 belongs to the Erv/ALR sulfhydryl oxidase family; the homologue proteins have been identified in all mitochondria-containing eukaryotes [12-14]. They all have a highly conserved catalytic domain of $\sim 100$ amino acids folded in a helix bundle and form stable dimers by noncovalently binding to flavin adenine dinucleotide (FAD) (Figure 1B) [15-17]. The catalytic (or FAD-binding) domain of yeast (Saccharomyces cerevisiae) Erv1 is at the $C$ terminus, containing a redox active site CXXC disulphide bond (Cys130-Cys133) and a CX16C structural disulphide (Cys159-Cys176) [18-20]. Yeast Erv1 also has a functionally important shuttle disulphide bond (Cys30-Cys33) in the non-conserved and unfolded $\mathrm{N}$-terminal domain, which is functionally important for interaction with Mia40 [18,20].

Erv/ALR enzymes can act as an oxidase by passing electrons to molecular oxygen and reducing oxygen to hydrogen peroxide, or as a cytochrome $c$ reductase by passing electrons to oxidized cytochrome $c$ (Figure 1A). Enzyme kinetic studies revealed that different Erv1/ALR enzymes have different oxidase and cytochrome $c$ reductase activities [17,21-23]. While both yeast Erv1 [23] and human ALR [21] seem to prefer cytochrome $c$ more than molecular oxygen as an electron acceptor, the ratio of relative cytochrome $c$ reductase to oxidase activity is quite different for these enzymes, with 15 for yeast Erv1 [23] and 107 for human ALR [21], as ALR uses oxygen poorly. On the other hand, yeast Erv2 [17] and avian QSOX [22] are good oxidases and readily reduce oxygen to hydrogen peroxide.

Mitochondria contain two membranes, the outer membrane (OM) and the inner membrane (IM), which are composed of phospholipid bilayers and proteins. The mitochondrial IMS is narrow and is almost filled with the cristae of the mitochondrial inner membrane, due to its large surface area. Biological reactions that take place in the IMS are surrounded by the mitochondrial IM and inevitably would be influenced by the membrane environment. Phosphatidylcholine (PC) and phosphatidylethanolamine (PE) are the major mitochondrial phospholipids in all cell types, which account for about $80 \%$ of total phospholipids. Cardiolipin is enriched in the mitochondrial IM of all organisms and may be confined to the IM, as has been argued for plants [24]. The majority of cardiolipin, almost $80 \%$, is located in the mitochondrial IM in yeast cells and constitutes about $20 \%$ 
of the membrane lipids found there [25]. Cardiolipin possesses a unique structure containing four (instead of two) fatty acid tails and two negatively charged phosphate groups, as shown in Figure 2A.

A

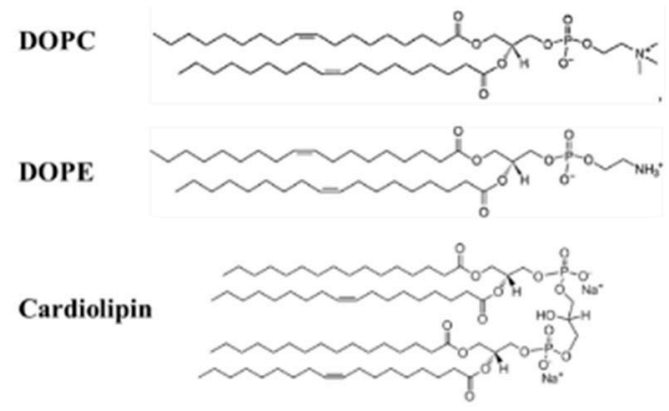

C

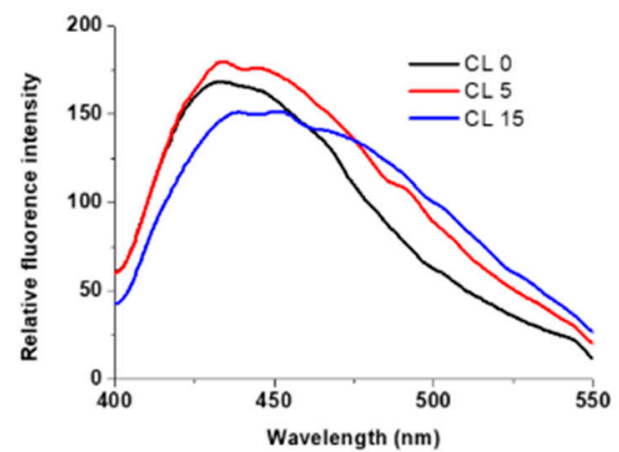

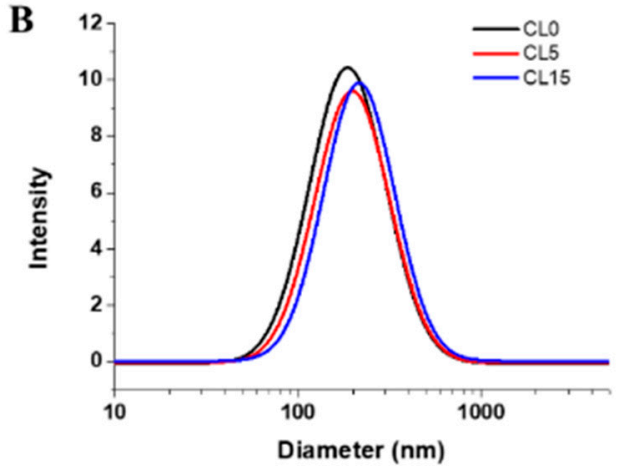

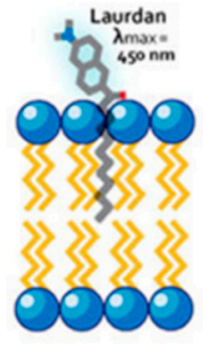

Figure 2. Characterization of liposomes. (A) Chemical structures of the lipids used for preparing liposomes. (B) Dynamic light scattering spectra of liposomes in the presence of $0 \%, 5 \%$, and $15 \%$ cardiolipin, respectively. (C) Fluorescence spectra of Laurdan in each formulation and a schematic model representing the insertion of Laurdan in liposomes. Experiments were performed with $100 \mu \mathrm{M}$ lipid and $1 \mu \mathrm{M}$ Laurdan. Lines represent liposome formulations CL0 (black), CL5 (red), CL15 (blue) respectively.

Although cytochrome $c$ is a water-soluble protein, it is membrane-associated. Studies have shown that about $85 \%$ of cytochrome $c$ is stored in the inner membrane cristae, and only $15-20 \%$ of total cytochrome $c$ is available in the IMS $[25,26]$. Cardiolipin is the preferential and major binding partner for cytochrome $c$ [27], and increasing cardiolipin in liposomes caused increased cytochrome $c$ binding [28]. Binding of cytochrome $c$ to membranes also led to an increase in peroxidase activity but was without effect on protein conformation or membrane penetration $[29,30]$. These findings suggest that the presence of membranes may play an important role in the folding and activity of these proteins.

Moreover, mitochondrial subfractionation analysis showed that about $50 \%$ of Erv1 [31] and ALR [32] are peripherally associated with the mitochondrial IM. However, whether this membrane association affects the folding, and, hence, function of these proteins, is unknown. Here, in this study, we investigated whether membrane association and the presence of cardiolipin play a role in mediating the oxidase and cytochrome $c$ reductase activities of Erv1, using liposomes as model bio-membranes to mimic the mitochondrial membrane. Our results showed that the presence of liposomes inhibits the cytochrome $c$ reductase activity of Erv1 strongly and in a cardiolipin concentration-dependent manner. The results of this study suggest that molecular oxygen is a better substrate than cytochrome $c$ for yeast Erv1 in the mitochondria. 


\section{Results}

\subsection{Preparation and Characterization of Liposomes}

The major components of the yeast mitochondrial OM are phosphatidylcholine $(\sim 45 \%)$, phosphatidylethanolamine $(\sim 23 \%)$, cardiolipin $(\sim 6 \%)$, and other lipids $(\sim 26 \%)$. For the IM, they are phosphatidylcholine (38\%), phosphatidylethanolamine 30\%), cardiolipin (20\%), and other lipids (12\%) [33]. The acyl chains of phospholipids of the yeast S. cerevisiae are mainly C16:1 and C18:1 with minor amounts of C14:0, C16:0, and C18:0 [34]. Since C16:1 lipids are not commercially available, liposomes with a lipid composition that resembles the mitochondrial membranes were prepared using C18:1 lipids, 1,2-dioleoyl-sn-glycero-3-phosphocholine (DOPC), and 1,2-dioleoyl-sn-glycero-3-phosphatidylethanolamine (DOPE) at a fixed ratio of 5:3 and in the presence of $0.5 \%$ or $15 \%$ of cardiolipin (Figure 2). We named these liposome formulations CL0, CL5, and CL15, accordingly, in this report. The average diameter of the liposomes in each formulation was measured using dynamic light scattering (Figure 2B) and was found to be the same, with or without the presence of cardiolipin. The mean diameter was $160 \pm 1 \mathrm{~nm}$, which was as expected, based on previous results [35].

Lipid packing is a crucial feature for liposomes, which can be analyzed using polarity-sensitive probes whose emission spectra depends on lipid packing. Laurdan, a microenvironment-sensitive fluorescent probe, was used to characterize the liposomes and assess whether the presence of cardiolipin affected the liposome packing, as described previously [27]. The fluorescence intensity of Laurdan at 440 and $490 \mathrm{~nm}$ (with excitation at $340 \mathrm{~nm}$ ) was sensitive to the ordered and disordered lipid phases, respectively [27]. As shown in Figure 2C, the fluorescence spectra of Laurdan in all three liposomes showed a dominant peak at $440 \mathrm{~nm}$. The overall spectra of Laurdan in CL0 and CL5 liposomes were very similar, suggesting that the lipids in both formulations were in a homogeneously ordered phase. However, a broad peak at around $490 \mathrm{~nm}$ appeared in the CL15 liposome spectrum, suggesting the presence of a disordered lipid phase. Thus, the general polarity (GP) of the liposomes was calculated based on: GP $=(\mathrm{I} 440-\mathrm{I} 490) /(\mathrm{I} 440+\mathrm{I} 490)$. The calculated GPs varied from 0.36 for CL0, 0.33 for CL5, and to 0.13 for CL15, indicating that the structure of the liposomes changed from an ordered phase toward a disordered phase. The polarity decreased by about $60 \%$ with the presence of $15 \%$ cardiolipin in CL15 compared with CL0, suggesting that cardiolipin significantly changes the structure of liposomes, possibly because cardiolipin is large in size, with four alkyl tails that disrupt the lipid organization.

\subsection{Effects of Liposomes on the Oxidase Activity of Erv1}

It was reported that approximately $50 \%$ Erv1 is peripherally associated with the mitochondrial IM [31]. However, whether the membrane association affects Erv1 folding and/or activity is unknown and unreported. Liposomes are a good model in which to study kinetic properties of enzymes in a membrane environment. Here, we investigated how the presence of the CL0, CL5, and CL15 liposome formulations affects the folding and oxidase activity of Erv1.

Erv1 is a FAD-binding protein with a signature UV-vis absorption spectrum including a peak maximum at $460 \mathrm{~nm}$. The absorption spectra of Erv1 in the absence and presence of the various liposome formulations were almost identical (Figure 3), suggesting the presence of these liposomes did not affect Erv1 FAD binding.

Next, the effects of the liposomes on the Erv1 oxidase activity were investigated using oxygen consumption analysis (Figure $4 \mathrm{~A}$ ), as described previously $[23,36]$. As shown at the beginning of Figure 4A, there was no oxygen consumption by DTT in the absence of Erv1. The oxygen consumption curves were differentiated, the oxygen consumption rate of oxygen against oxygen concentration was plotted, and the enzyme kinetic parameters $\mathrm{K}_{\mathrm{m}}$ and $\mathrm{k}_{\mathrm{cat}}$ were estimated (Figure $4 \mathrm{~B}$, Table 1 ). The data showed that addition of liposomes decreased both $\mathrm{K}_{\mathrm{m}}$ and $\mathrm{k}_{\mathrm{cat}}$ to a similar level (Figure $4 \mathrm{C}$ ). Overall, the relative oxidase catalytic activity of Erv1 was reduced to about $70 \%$ of the control (in the absence 
of liposomes) in all three cases (Figure 4D). Thus, the presence of liposomes suppressed the oxidase activity of Erv1 partially, regardless of cardiolipin content, suggesting that cardiolipin was not a specific binding partner for Erv1.

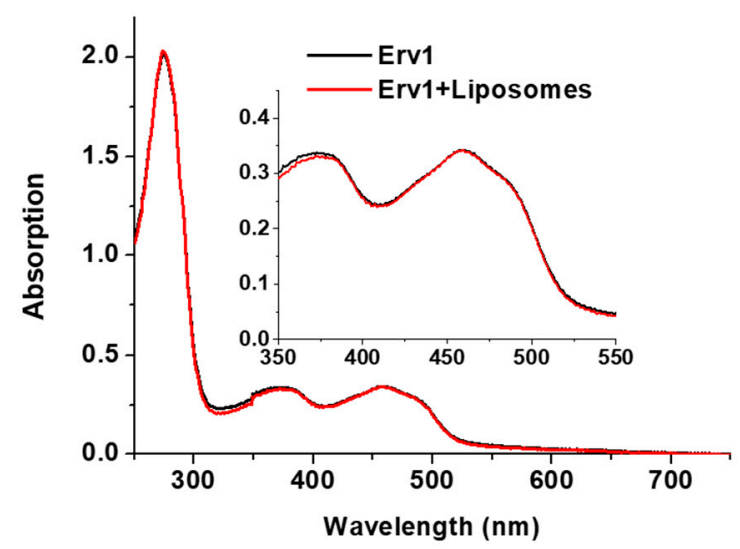

Figure 3. UV-vis absorption spectra of Erv1 in the absence (black) and presence (red) of $0.5 \mathrm{mM}$ CL15 liposomes. The same overlapping spectra were obtained for Erv1 in the presence of CL0 or CL5 liposomes (data not shown). The molar ratio of lipids:Erv1 was 60:1.

A
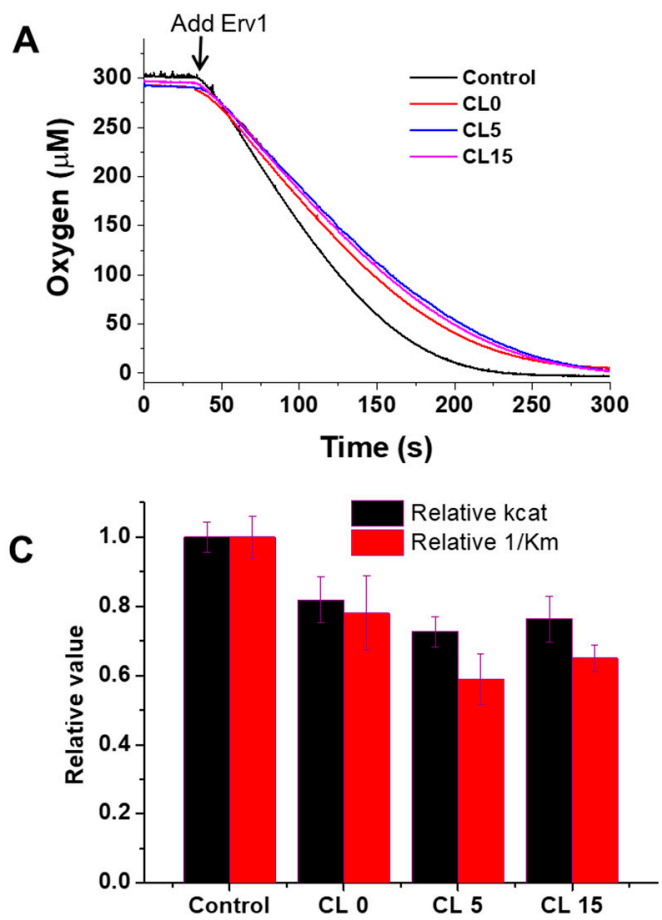

B
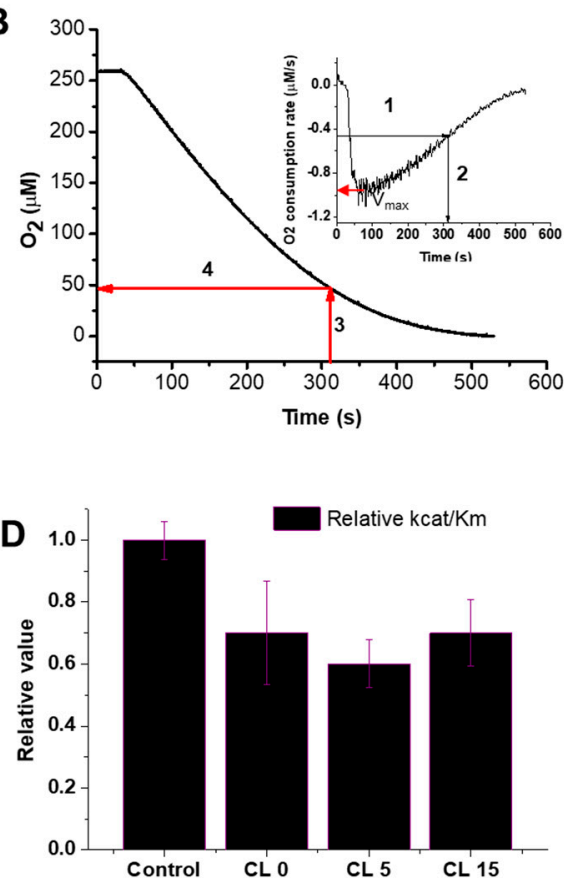

Relative $\mathrm{kcat} / \mathrm{Km}$

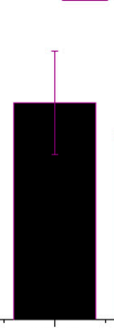

CL 0

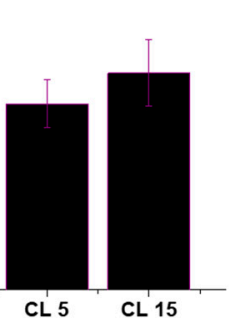

Figure 4. Effects of liposomes on the oxidase activity of Erv1. (A) Time course of oxygen consumption by $5 \mathrm{mM}$ DTT catalyzed by $5 \mu \mathrm{M}$ Erv1, in the absence (Control) and presence of $0.5 \mathrm{mM}$ liposomes CL0, CL5, and CL15, respectively. The molar ratio of lipids:Erv1 was 100:1. (B) An example of how the oxygen consumption curve and the first derivative plot (insert) were used to identify the time point at which $50 \%$ of the maximal velocity $\left(\mathrm{V}_{\max }\right)$ was reached; this was then used for $\mathrm{K}_{\mathrm{m}}$ estimation. (C) The relative oxidase $\mathrm{k}_{\text {cat }}$ (black) and $1 / \mathrm{K}_{\mathrm{m}}$ (red) of Erv1. (D) Relative oxidase catalytic efficiency of Erv1. Control: without liposomes. CL0, CL5, and CL15: $0.5 \mathrm{mM}$ liposomes. The error bars represent the standard error of the mean (SEM), $n=3$; differences between each result and the corresponding controls were statistically analyzed using Kruskal-Wallis (one-way ANOVA) test, all $p<0.05$. 
Table 1. Summary of the kinetic parameters determined in various conditions, at $25^{\circ} \mathrm{C}, \mathrm{pH} 7.4$.

\begin{tabular}{ccccc}
\hline Substrate & Liposomes & $\mathbf{k}_{\mathbf{c a t}}\left(\mathbf{s}^{-\mathbf{1}}\right)$ & $\mathbf{K}_{\mathbf{m}}(\boldsymbol{\mu M})$ & $\mathbf{k}_{\mathbf{c a t}} / \mathbf{K}_{\mathbf{m}}\left(\mathbf{M}^{-\mathbf{1}} \mathbf{s}^{-\mathbf{1}}\right)$ \\
\hline \multirow{3}{*}{ Oxygen } & $\begin{array}{c}\text { Control } \\
\text { (No liposomes) }\end{array}$ & $1.0 \pm 0.1$ & $50 \pm 10$ & $2.0 \times 10^{4}(100 \%)$ \\
\cline { 2 - 5 } & CL0 & $0.82 \pm 0.02$ & $60.0 \pm 6$ & $1.4 \times 10^{4}(70 \%)$ \\
\cline { 2 - 5 } & CL5 & $0.74 \pm 0.01$ & $58.8 \pm 6$ & $1.2 \times 10^{4}(60 \%)$ \\
\hline \multirow{2}{*}{ Cytochrome $c$} & $\begin{array}{c}\text { CL15 } \\
\text { Control }\end{array}$ & $0.76 \pm 0.02$ & $53.2 \pm 5$ & $1.4 \times 10^{4}(70 \%)$ \\
\cline { 2 - 5 } & CL0 & $0.75 \pm 0.03$ & $52 \pm 4$ & $1.4 \times 10^{4}(39 \%)$ \\
\cline { 2 - 5 } & CL5 & $0.61 \pm 0.04$ & $74 \pm 3$ & $8.2 \times 10^{3}(23 \%)$ \\
\cline { 2 - 5 } & CL15 & $0.48 \pm 0.02$ & $105 \pm 7$ & $4.6 \times 10^{3}(13 \%)$ \\
\hline
\end{tabular}

\subsection{Effects of Liposomes on Cytochrome $c$ Reductase Activity of Erv1}

Since cytochrome $c$ is closely associated with the mitochondrial IM, whether heme binding to cytochrome $c$ was affected by liposomes was assessed first. For this, UV-vis absorption spectra were recorded in the presence and absence of the liposomes. As shown in Figure 5A, the spectra were almost the same, suggesting cofactor binding to cytochrome $c$ was unaffected and no conformational changes were caused by the liposomes.

A
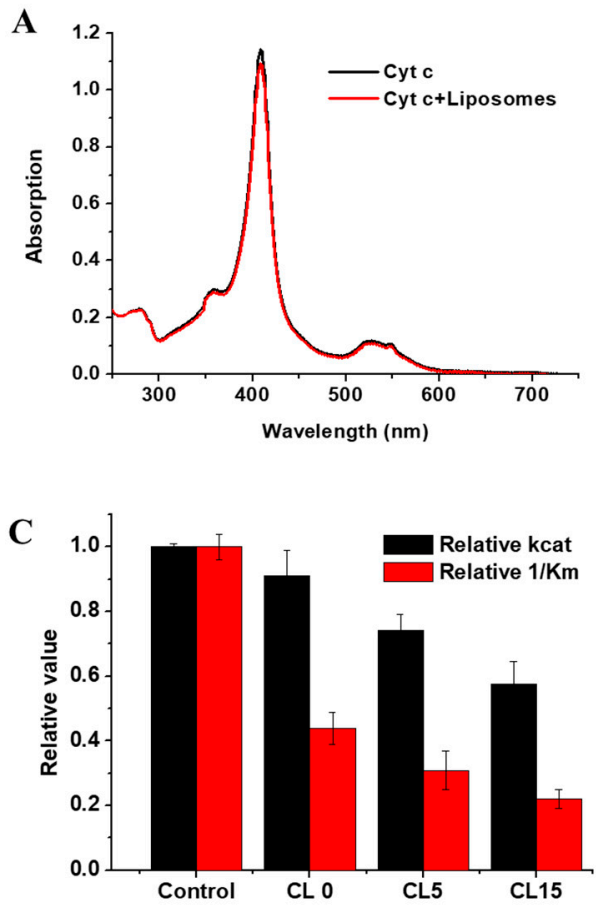

B

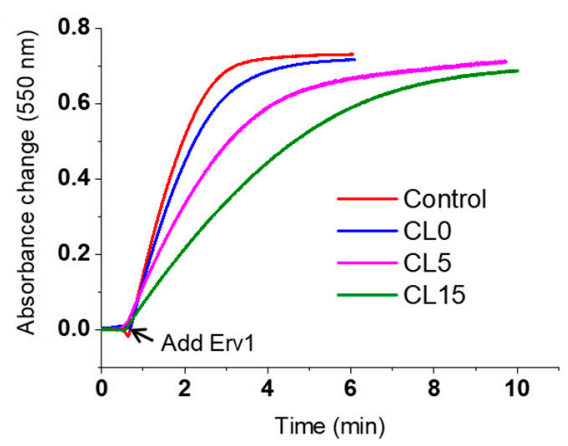

D

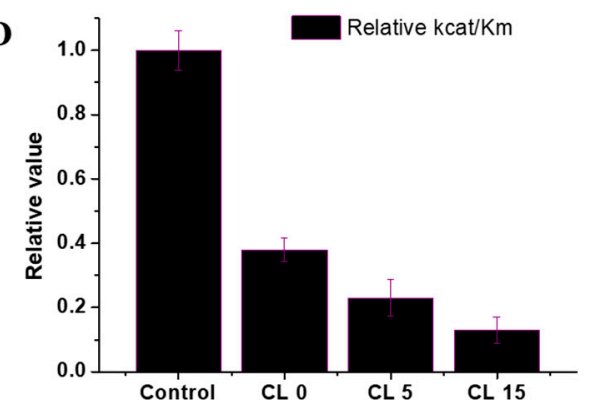

Figure 5. Effects of liposomes on the cytochrome $c$ reductase activity of Erv1. (A) Absorption spectra of cytochrome $c$ in the absence and presence of CL15 liposomes. The same spectra were obtained with CL0 and CL5 liposomes (data not shown) (B) Time course of cytochrome $c$ reduction, followed by absorbance at $550 \mathrm{~nm}$. (C) Relative enzyme kinetic parameters of cytochrome $c$ reductase activity, $\mathrm{k}_{\text {cat }}$ (black) and relative $1 / \mathrm{K}_{\mathrm{m}}$ (red). (D) The relative cytochrome $c$ reductase catalytic efficiency of Erv1. Control: the reaction without liposomes. DTT $(1 \mathrm{mM})$ and liposomes $(0.5 \mathrm{mM})$ were used in all cases. The error bars represent the SEM, $n=3$; differences between each result and the corresponding controls were statistically analyzed using Kruskal-Wallis (one-way ANOVA) test, all $p<0.001$. 
Next, we investigated whether the presence of liposomes affects the cytochrome $c$ reductase activity of Erv1, using DTT as an electron donor and cytochrome $c$ as an acceptor, as established previously for Mia40 [18,23]. Time courses of cytochrome $c$ absorbance change at $550 \mathrm{~nm}$ were measured in the absence and presence of various liposome formulations, which showed that the absorbance change was decreased by the presence of liposomes and was dependent on cardiolipin concentration (Figure 5B). In all cases, the rate of non-enzyme catalyzed cytochrome $c$ reduction by DTT in the absence of Erv1 was negligible. The absorbance changes were analyzed by differentiation, and the enzyme parameters were calculated (Figure 5C, Table 1). While the initial rate of cytochrome $c$ reduction was decreased to about $90 \%$ in the presence of the CL0 formulation, it was decreased further to about $74 \%$ and $58 \%$ of that of the control (without liposomes) in the presence of CL5 or CL15 liposomes (Figure 5C). The overall catalytic efficiency $\left(\mathrm{k}_{\text {cat }} / \mathrm{K}_{\mathrm{m}}\right)$ of cytochrome $c$ was reduced to $40-10 \%$ in the presence of CL0-CL15 (Figure 5D, Table 1).

Taken together, our results showed that the liposomes have a strong inhibitory effect on the cytochrome $c$ reductase activity of Erv1 and that this inhibition increased with the increasing cardiolipin content. With the presence of $15 \%$ cardiolipin in the liposome, a concentration similar to that observed in the mitochondrial IM, the cytochrome $c$ reductase activity of Erv1 was reduced to about $10 \%$ that of the control (in the absence of liposomes). This was largely due to the effect of CL15 on $\mathrm{K}_{\mathrm{m}}$, the binding of cytochrome $c$ to Erv1.

\section{Discussion}

In this study, we investigated how liposomes and cardiolipin affect the oxidase and cytochrome $c$ reductase activities of mitochondrial Erv1. It was reported that about 50\% Erv1 was peripherally associated with the mitochondrial IM [31]. The overlapping spectra and slightly decreased oxidase activity of Erv1 at the molar ratio of lipids:Erv1 between 60:1 to 100:1 suggested that Erv1 was probably not bound to liposomes. Considering the high abundance of proteins in mitochondrial IMS and the localization of Erv1's partner protein Mia40, the membrane association of Erv1 in mitochondria is most likely due to its interaction with Mia40, rather than lipids.

Unlike the oxidase activity, the liposomes had a much clearer impact on the cytochrome $c$ catalytic efficiency of Erv1, and the inhibitory effect increased with the increased cardiolipin content of the liposomes (Figure 5). Our results suggested that cardiolipin, PC, and PE inhibit the cytochrome $c$ reductase activity of Erv1 by affecting the interactions between Erv1 and cytochrome $c$ since the $K_{m}$ increased with the presence of the liposomes and in a cardiolipin concentration-dependent manner. Cardiolipin was predominantly responsible for the inhibitory effect. Cardiolipin has four acyl chains, equivalent to two PC or PE molecules (see Figure 1A). Furthermore, there are two negative charges on the head group of cardiolipin, which favors electrostatic attractions with the highly positive-charged cytochrome $c$, which has a pI of about 9. Consequently, the interactions between Erv1 and cytochrome $c$ weakened, as evidenced by the 4.6-fold increase in $\mathrm{K}_{\mathrm{m}}$, compared with the $\mathrm{K}_{\mathrm{m}}$ of the control and CL15 (Table 1). Moreover, the addition of cardiolipin changed the liposome structure from an ordered to a more disordered phase in CL15. Thus, we would predict that CL15 liposomes would have a less rigid structure than orderly packed liposomes, which may enhance cristae formation and favor the interaction of cytochrome $c$ with the liposomes or mitochondria IM. Hence, the interactions or communications between cytochrome $c$ and Erv1 were weakened or partially reduced.

The presence of liposomes, such as CL15, decreased the oxidase activity of Erv1 by about $30 \%$, to about $70 \%$ of the catalytic efficiency of Erv1 in the absence of liposomes. However, the CL15 formulation inhibited about $90 \%$ of the cytochrome $c$ reductase activity of Erv1 (Figures 4 and 5, Table 1). We showed that, in the absence of liposomes, the cytochrome $c$ reductase activity of Erv1 was slightly higher than that of the oxidase activity, with a relative substrate specificity (RSS), defined as the ratio of $\left(\mathrm{k}_{\mathrm{cat}} / \mathrm{K}_{\mathrm{m}}\right)^{\text {Cyt c }}$ to $\left(\mathrm{k}_{\mathrm{cat}} / \mathrm{K}_{\mathrm{m}}\right)^{\text {Oxygen }}$ between 2-15, depending on the experimental conditions [23] (Table 1). In this study, our results showed that in the presence of CL15 liposomes, the RSS ratio is about 0.33 (Table 1). Thus, molecular oxygen is a better substrate than cytochrome $c$ in the presence 
of CL15 liposomes. Since CL15 liposomes mimic the mitochondria IM, this result indicates that molecular oxygen may be also a better substrate than cytochrome $c$ in the mitochondrial IMS in yeast. Consistent with this, a previous study showed that the apparent $K_{m}$ of the GSH-Mia40-Erv1 system for oxygen is very low $(\sim 3 \mu \mathrm{M})$ [23]. About $15 \%$ of mitochondrial cytochrome $c$ is tightly bound to the cristae of the mitochondrial IM and about $85 \%$ of cytochrome $c$ is accessible in the bulk solution [37]. However, the concentration of the oxidized cytochrome $c$ in the mitochondrial IMS that is available for Erv1 is unknown and it may vary widely depending on cell growth conditions. The results of this study support that molecular oxygen is a better substrate than cytochrome $c$ for yeast Erv1 in the mitochondria.

The oxidase activity of Erv1 produces two molecules of hydrogen peroxide $\left(\mathrm{H}_{2} \mathrm{O}_{2}\right)$ for every one molecule of $\mathrm{O}_{2}$ consumed. Hydrogen peroxide is considered a reactive oxygen species (ROS), which, at high levels, can damage all biomolecules and cause oxidative stress. Oxidative stress has been linked to many diseases and to the process of aging [38]. On the other hand, ROS produced under normal physiological conditions play an important regulatory role in cellular metabolic processes, and mitochondria are the main endogenous source of cellular ROS, as a result of incomplete electron transfer along the electron transport chain (ETC) [39]. To avoid $\mathrm{H}_{2} \mathrm{O}_{2}$ accumulation and potential oxidative stress caused by Erv1, yeast has an enzyme, cytochrome $c$ peroxidase (Ccp1), in the mitochondrial IMS, which can effectively remove the $\mathrm{H}_{2} \mathrm{O}_{2}$ produced by Erv1 [11]. Under oxidative stress conditions, $\mathrm{H}_{2} \mathrm{O}_{2}$ can also be removed by the IMS-localized glutathione peroxidase Gpx3 [40]. The importance of the Erv1/ALR enzymes was also demonstrated by the identification of a disease directly related to a single mutation in ALR, the human homologue of Erv1 [41]. A single arginine-to-histidine substitution (R194H) in the C-terminus of ALR was identified in children with autosomal recessive myopathy; the effects included respiratory chain deficiency, abnormal mitochondrial morphology, and increased accumulation of mitochondrial DNA (mtDNA) deletions, a temperature-sensitive growth phenotype and reduced cytochrome $c$ oxidase (complex IV) activity [41]. Protein characterization studies showed that the mutation results in decreased FAD-binding and thermal stability of the protein, and a mild effect on its function in vitro in the absence of liposomes [20,42]. A study showed that the human short form of ALR exhibited a higher cytochrome $c$ reductase activity than its oxidase activity in the absence of liposomes, compared with yeast Erv1 [21]. Moreover, no yeast homologue of $\mathrm{Ccp} 1$ has been identified in human. It will, therefore, be interesting to interrogate how liposomes and cardiolipin affect the function of human ALR and, more importantly, the physiological effects. The results of this study showed that molecular oxygen is a suitable substrate for the MIA pathway in yeast. Thus, it will be interesting to understand whether the resulting hydrogen peroxide plays a role in mitochondria-nuclear communication linking mitochondrial oxidative stress status and expression of nuclear DNA-encoded mitochondrial proteins together.

\section{Materials and Methods}

All chemicals used in this study were analytical grade and were from Sigma or Fisher, unless specified. All solutions were prepared using MilliQ water. All experiments were carried out in a Tris buffer ( $50 \mathrm{mM}$ Tris- $\mathrm{HCl}, 150 \mathrm{mM} \mathrm{NaCl}, 1 \mathrm{mM}$ EDTA, pH 7.4) unless specified.

\subsection{Protein Expression and Purification}

The ERV1 gene was cloned using a pET-24a(+) plasmid and expressed in Escherichia coli Rosetta-gamiTM2 cells (Novagen). Cells were grown in Luria broth medium (Formedium Ltd.) containing $50 \mu \mathrm{g} / \mathrm{mL}$ of kanamycin (Fisher Scientific) at $37^{\circ} \mathrm{C}$, until the OD600 reached 0.6. Then, proteins were induced by addition of $0.5 \mathrm{mM}$ isopropyl $\beta$-D- 1 thiogalactopyranoside (IPTG) at $16^{\circ} \mathrm{C}$ and were incubated overnight $(16 \mathrm{~h})$. Cells were collected by centrifugation $\left(4^{\circ} \mathrm{C}, 5000 \times \mathrm{g} \mathrm{rpm}, 20 \mathrm{~min}\right)$.

Collected cells were resuspended in binding buffer $(20 \mathrm{mM}$ imidazol, $50 \mathrm{mM}$ Tris- $\mathrm{HCl}$, $150 \mathrm{mM} \mathrm{NaCl}, \mathrm{pH} 7.4$ ) to $\sim 0.2 \mathrm{mg} / \mathrm{mL}$ with $100 \mu \mathrm{M}$ FAD and two EDTA-free protease inhibitor cocktail tablets (Roche). The cell membranes were ruptured by sonication (10/50 s ON/OFF, $30 \%$ amplitude, 
$50 \mathrm{~min})$ and the cell debris and soluble proteins were separated by centrifugation $\left(4{ }^{\circ} \mathrm{C}, 17,000 \mathrm{rpm}\right.$, $60 \mathrm{~min}$ ). The supernatant containing the majority of the expressed proteins was carefully collected and filtered with a $0.45-\mu \mathrm{m}$ filter. Then, the supernatant was purified following a standard protocol for His-tagged protein purification. (1) Firstly, the supernatant was applied to a column packed with a 2-mL volume of $\mathrm{Ni}^{2+}$ charged $\mathrm{Hi} \mathrm{Ni}-\mathrm{NTA}\left(\mathrm{Ni}^{2+}\right.$-nitrilotriacetate) His-Bind beads (Novagen). (2) Following binding of the target proteins, the beads were washed with $10 \mathrm{~mL}$ binding buffer. (3) The protein-bound beads were then washed with $20 \mathrm{~mL}$ of washing buffer $(40 \mathrm{mM}$ imidazol, $50 \mathrm{mM}$ Tris- $\mathrm{HCl}, 150 \mathrm{mM} \mathrm{NaCl}, \mathrm{pH}$ 7.4). (4) The target proteins were eluted by washing with $6 \mathrm{~mL}$ elution buffer (500 mM imidazol, $50 \mathrm{mM}$ Tris- $\mathrm{HCl}, 150 \mathrm{mM} \mathrm{NaCl}, \mathrm{pH} 7.4)$ and stored in an excess of FAD at $-80{ }^{\circ} \mathrm{C}$ for preservation.

Cytochrome $c$ (cat.9007-43-6) was purchased from Sigma and its redox state was checked, based on absorption at $550 \mathrm{~nm}$; more than $95 \%$ of the proteins were in their oxidized form and used as purchased.

\subsection{Liposome Preparation}

Liposomes were prepared by dissolving 1,2-dioleoyl-sn-glycero-3-phosphocholine (DOPC; $500 \mu \mathrm{mol} / \mathrm{L}$ ), 1,2-dioleoyl-sn-glycero-3-phosphocholine (DOPE; $300 \mu \mathrm{mol} / \mathrm{L}$ ), and varied concentration of cardiolipin $(0,50,150 \mu \mathrm{mol} / \mathrm{L})$ in chloroform to mimic the molecular ratio of the mitochondria IM (PC 50\%: PE 30\%: CL 15\%). Solvent was removed by rotary evaporation to produce a thin lipid film, which was dried in a vacuum oven overnight. The lipid mixtures were rehydrated with Tris buffer (50 mM Tris-HCl, $150 \mathrm{mM} \mathrm{NaCl}, 1 \mathrm{mM}$ EDTA, pH 7.4) to yield a lipid concentration of $1 \mathrm{mM}$. The resulting suspension was extruded 11 times using a 1-mL Mini-Extruder (Avanti Polar Lipids) through a $0.1-\mu \mathrm{m}, 19-\mathrm{mm}$ polycarbonate membrane, surrounded by two $10-\mathrm{mm}$ filter supports, in order to produce a unilamellar liposome formulation. The suspension was stored at $4{ }^{\circ} \mathrm{C}$ until use. The size (hydrodynamic diameter) distributions were measured by dynamic light scattering (DLS).

\subsection{UV-Visible Spectroscopy}

Absorption spectra were recorded from 250 to $700 \mathrm{~nm}$, at 1-nm intervals, in a 1-cm path length quartz cuvette using a Cary 300 Bio UV-Visible spectrophotometer (Varian). An extinction coefficient of $12.3 \mathrm{mM}^{-1} \mathrm{~cm}^{-1}$ at $460 \mathrm{~nm}$ was used to calculate concentration of Erv1, and $\varepsilon 450 \mathrm{~nm}$ of $11.3 \mathrm{mM}^{-1} \mathrm{~cm}^{-1}$ was used for free FAD. An extinction coefficient $\varepsilon 550 \mathrm{~nm}$ of $29.5 \mathrm{mM}^{-1} \mathrm{~cm}^{-1}$ for oxidized cytochrome $c$, and $8.4 \mathrm{mM}^{-1} \mathrm{~cm}^{-1}$ for reduced cytochrome $c$ were used.

\subsection{Oxygen Consumption Assays}

Erv1 enzymatic activity toward oxygen was measured using a Clark-type oxygen electrode (Hansatech Instruments) in $0.5 \mathrm{~mL}$ of reaction volume at $25{ }^{\circ} \mathrm{C}$ in Tris buffer ( $50 \mathrm{mM}$ Tris- $\mathrm{HCl}$, $150 \mathrm{mM} \mathrm{NaCl}, 1 \mathrm{mM}$ EDTA, pH 7.4), as previously described [21]. When DTT was used as an electron donor, bovine erythrocyte superoxide dismutase 1 (SOD) (Sigma) was added at 10 units/mL to exclude the potential interference of superoxide ions. For Erv1 oxidase kinetic parameter determination, $5 \mathrm{mM}$ DTT was used, so that the DTT concentration was more than 10-fold in excess of the $\mathrm{O}_{2}$ concentration $(\sim 250 \mu \mathrm{M})$. The initial slope of the oxygen consumption curve was used to represent the oxygen consumption rate and was calculated by data differentiation using OriginPro software. At least three independent experimental repeats were performed for each experiment. Data were shown as mean \pm SEM $(n=3)$, and differences between groups were statistically analyzed using a Kruskal-Wallis (one-way ANOVA) test.

\subsection{Cytochrome c Reduction Assay}

The cytochrome $c$ reduction assay was performed using a Cary 300 Bio UV-Visible spectrophotometer (Varian). Reduction rate was measured via the absorbance change of cytochrome $c$ at $550 \mathrm{~nm}$. An extinction coefficient $\varepsilon 550 \mathrm{~nm}$ of $29.5 \mathrm{mM}^{-1} \mathrm{~cm}^{-1}$ for oxidized cytochrome $c$ and $8.4 \mathrm{mM}^{-1} \mathrm{~cm}^{-1}$ for reduced cytochrome $c$ were used. The reduction rate of cytochrome $c$ was 
calculated using a $\Delta \varepsilon 550$ of $21.1 \mathrm{mM}^{-1} \mathrm{~cm}^{-1}$, the extinction coefficient difference between reduced and oxidized cytochrome $c$. The rate of non-enzyme catalyzed cytochrome $c$ reduction by DTT was measured in the absence of Erv1, which was used to calculate the background rate and was subtracted from the rate of reduction, in the presence of Erv1. The data were analyzed using OriginPro software. At least three experimental repeats were performed for all experiments. Data were shown as mean $\pm \operatorname{SEM}(n=3)$, and differences between groups were statistically analyzed using a Kruskal-Wallis (one-way ANOVA) test.

Author Contributions: Conceptualization, X.T. and H.L.; methodology, X.T., L.K.H., and H.L.; formal analysis, X.T. and H.L.; investigation, X.T.; resources, X.T., L.K.H., and H.L.; writing-original draft preparation, X.T.; writing —review and editing, X.T., H.L., and L.K.H.; supervision, H.L.; project administration, H.L.; funding acquisition, H.L. All authors have read and agreed to the published version of the manuscript.

Funding: This research received no external funding.

Acknowledgments: We thank Chinese Scholarship Council (CSC) studentship support for X.T. and Ping Xiao of the University of Manchester for student co-supervision and materials support.

Conflicts of Interest: The authors declare no conflict of interest. The funders had no role in the design of the study; in the collection, analyses, or interpretation of data; in the writing of the manuscript; or in the decision to publish the results.

\section{Abbreviations}

$\begin{array}{ll}\text { ALR } & \text { Augmenter of liver regeneration } \\ \text { DTT } & \text { Dithiothreitol } \\ \text { DOPC } & \text { 1,2-dioleoyl-sn-glycero-3-phosphocholine } \\ \text { DOPCE } & \text { 1,2-dioleoyl-sn-glycero-3-phosphatidylethanolamine } \\ \text { Erv } & \text { Essential for respiration and viability } \\ \text { FAD } & \text { Flavin adenine dinucleotide } \\ \text { IMS } & \text { Intermembrane space } \\ \text { IM } & \text { Inner membrane } \\ \text { MIA } & \text { Mitochondrial import and assembly } \\ \text { OM } & \text { Outer membrane }\end{array}$

\section{References}

1. Neupert, W. Protein import into mitochondria. Annu. Rev. Biochem. 1997, 66, 863-917. [CrossRef] [PubMed]

2. Herrmann, J.M.; Hell, K. Chopped, trapped or tacked-protein translocation into the IMS of mitochondria. Trends Biochem. Sci. 2005, 30, 205-212. [CrossRef]

3. Stojanovski, D.; Bragoszewski, P.; Chacinska, A. The MIA pathway: A tight bond between protein transport and oxidative folding in mitochondria. Biochim. Biophys. Acta 2012, 1823, 1142-1150. [CrossRef] [PubMed]

4. Hell, K. The Erv1-Mia40 disulfide relay system in the intermembrane space of mitochondria. Biochim. Biophys. Acta 2008, 1783, 601-609. [CrossRef]

5. MacPherson, L.; Tokatlidis, K. Protein trafficking in the mitochondrial intermembrane space: Mechanisms and links to human disease. Biochem. J. 2017, 474, 2533-2545. [CrossRef] [PubMed]

6. Lu, H.; Allen, S.; Wardleworth, L.; Savory, P.; Tokatlidis, K. Functional TIM10 chaperone assembly is redox-regulated in vivo. J. Biol. Chem. 2004, 279, 18952-18958. [CrossRef]

7. Mesecke, N.; Terziyska, N.; Kozany, C.; Baumann, F.; Neupert, W.; Hell, K.; Herrmann, J.M. A disulfide relay system in the intermembrane space of mitochondria that mediates protein import. Cell 2005, 121, 1059-1069. [CrossRef]

8. Ceh-Pavia, E.; Tang, X.; Liu, Y.; Heyes, D.J.; Zhao, B.; Xiao, P.; Lu, H. Redox characterisation of Erv1, a key component for protein import and folding in yeast mitochondria. FEBS J. 2020, 287, 2281-2291. [CrossRef]

9. Allen, S.; Balabanidou, V.; Sideris, D.P.; Lisowsky, T.; Tokatlidis, K. Erv1 mediates the Mia40-dependent protein import pathway and provides a functional link to the respiratory chain by shuttling electrons to cytochrome c. J. Mol. Biol. 2005, 353, 937-944. [CrossRef]

10. Herrmann, J.M.; Köhl, R. Catch me if you can! Oxidative protein trapping in the intermembrane space of mitochondria. J. Cell Biol. 2007, 176, 559-563. [CrossRef] 
11. Dabir, D.V.; Leverich, E.P.; Kim, S.K.; Tsai, F.D.; Hirasawa, M.; Knaff, D.B.; Koehler, C.M. A role for cytochrome $c$ and cytochrome $c$ peroxidase in electron shuttling from Erv1. EMBO J. 2007, 26, 4801-4811. [CrossRef] [PubMed]

12. Hagiya, M.; Francavilla, A.; Polimeno, L.; Ihara, I.; Sakai, H.; Seki, T.; Shimonishi, M.; Porter, K.A.; Starzl, T.E. Cloning and sequence analysis of the rat augmenter of liver regeneration (ALR) gene: Expression of biologically active recombinant ALR and demonstration of tissue distribution. Proc. Natl. Acad. Sci. USA 1994, 91, 8142-8146. [CrossRef] [PubMed]

13. Levitan, A.; Danon, A.; Lisowsky, T. Unique features of plant mitochondrial sulfhydryl oxidase. J. Biol. Chem. 2004, 279, 20002-20008. [CrossRef] [PubMed]

14. Eckers, E.; Petrungaro, C.; Gross, D.; Riemer, J.; Hell, K.; Deponte, M. Divergent Molecular Evolution of the Mitochondrial Sulfhydryl: Cytochrome $c$ Oxidoreductase Erv in Opisthokonts and Parasitic Protists. J. Biol. Chem. 2013, 288, 2676-2688. [CrossRef] [PubMed]

15. Gross, E.; Sevier, C.S.; Vala, A.; Kaiser, C.A.; Fass, D. A new FAD-binding fold and intersubunit disulfide shuttle in the thiol oxidase Erv2p. Nat. Struct. Biol. 2002, 9, 61-67. [CrossRef]

16. Guo, P.C.; Ma, J.D.; Jiang, Y.L.; Wang, S.J.; Bao, Z.Z.; Yu, X.J.; Chen, Y.X.; Zhou, C.Z. Structure of Yeast Sulfhydryl Oxidase Erv1 Reveals Electron Transfer of the Disulfide Relay System in the Mitochondrial Intermembrane Space. J. Biol. Chem. 2012, 287, 34961-34969. [CrossRef]

17. Wang, W.Z.; Winther, J.R.; Thorpe, C. Erv2p: Characterization of the redox behavior of a yeast sulfhydryl oxidase. Biochemistry 2007, 46, 3246-3254. [CrossRef]

18. Ang, S.K.; Lu, H. Deciphering Structural and Functional Roles of Individual Disulfide Bonds of the Mitochondrial Sulfhydryl Oxidase Erv1p. J. Biol. Chem. 2009, 284, 28754-28761. [CrossRef]

19. Hofhaus, G.; Lee, J.E.; Tews, I.; Rosenberg, B.; Lisowsky, T. The N-terminal cysteine pair of yeast sulfhydryl oxidase Erv1p is essential for in vivo activity and interacts with the primary redox centre. Eur. J. Biochem. 2003, 270, 1528-1535. [CrossRef]

20. Ceh-Pavia, E.; Ang, S.K.; Spiller, M.P.; Lu, H. The disease-associated mutation of the mitochondrial thiol oxidase Erv1 impairs cofactor binding during its catalytic reaction. Biochem. J. 2014, 464, 449-459. [CrossRef]

21. Farrell, S.R.; Thorpe, C. Augmenter of Liver Regeneration: A Flavin-Dependent Sulfhydryl Oxidase with Cytochrome $c$ Reductase Activity. Biochemistry 2005, 44, 1532-1541. [CrossRef] [PubMed]

22. Hoober, K.L.; Thorpe, C. Egg white sulfhydryl oxidase: Kinetic mechanism of the catalysis of disulfide bond formation. Biochemistry 1999, 38, 3211-3217. [CrossRef] [PubMed]

23. Tang, X.; Ang, S.K.; Ceh-Pavia, E.; Heyes, D.J.; Lu, H. Kinetic characterisation of Erv1, a key component for protein import and folding in yeast mitochondria. FEBS J. 2020, 287, 1220-1231. [CrossRef] [PubMed]

24. Harwood, J.L. Phosphoglycerides of mitochondrial membranes. In Methods in Enzymology; Academic Press: Cambridge, MA, USA, 1987; Volume 148, pp. 475-485.

25. Scorrano, L.; Ashiya, M.; Buttle, K.; Weiler, S.; Oakes, S.A.; Mannella, C.A.; Korsmeyer, S.J. A Distinct Pathway Remodels Mitochondrial Cristae and Mobilizes Cytochrome $c$ during Apoptosis. Dev. Cell 2002, 2, 55-67. [CrossRef]

26. Bernardi, P.; Azzone, G.F. Cytochrome $c$ as an electron shuttle between the outer and inner mitochondrial membranes. J. Biol. Chem. 1981, 256, 7187-7192.

27. Suga, K.; Hamasaki, A.; Chinzaka, J.; Umakoshi, H. Liposomes modified with cardiolipin can act as a platform to regulate the potential flux of NADP+-dependent isocitrate dehydrogenase. Metab. Eng. Commun. 2016, 3, 8-14. [CrossRef]

28. Rytömaa, M.; Kinnunen, P.K. Evidence for two distinct acidic phospholipid-binding sites in cytochrome $c$. J. Biol. Chem. 1994, 269, 1770-1774.

29. Ascenzi, P.; Coletta, M.; Wilson, M.T.; Fiorucci, L.; Marino, M.; Polticelli, F.; Sinibaldi, F.; Santucci, R. Cardiolipin-cytochrome $c$ complex: Switching cytochrome $c$ from an electron-transfer shuttle to a myoglobinand a peroxidase-like heme-protein. IUBMB Life 2015, 67, 98-109. [CrossRef]

30. Mandal, A.; Hoop, C.L.; DeLucia, M.; Kodali, R.; Kagan, V.E.; Ahn, J.; van der Wel, P.C.A. Structural Changes and Proapoptotic Peroxidase Activity of Cardiolipin-Bound Mitochondrial Cytochrome c. Biophys. J. 2015, 109, 1873-1884. [CrossRef]

31. Stojanovski, D.; Milenkovic, D.; Müller, J.M.; Gabriel, K.; Schulze-Specking, A.; Baker, M.J.; Ryan, M.T.; Guiard, B.; Pfanner, N.; Chacinska, A. Mitochondrial protein import: Precursor oxidation in a ternary complex with disulfide carrier and sulfhydryl oxidase. J. Cell Biol. 2008, 183, 195-202. [CrossRef] 
32. Sztolsztener, M.E.; Brewinska, A.; Guiard, B.; Chacinska, A. Disulfide Bond Formation: Sulfhydryl Oxidase ALR Controls Mitochondrial Biogenesis of Human MIA40. Traffic 2013, 14, 309-320. [CrossRef] [PubMed]

33. Daum, G.; Vance, J.E. Import of lipids into mitochondria. Prog. Lipid Res. 1997, 36, 103-130. [CrossRef]

34. Wagner, S.; Paltauf, F. Generation of glycerophospholipid molecular species in the yeast Saccharomyces cerevisiae. Fatty acid pattern of phospholipid classes and selective acyl turnover at sn-1 and sn-2 positions. Yeast 1994, 10, 1429-1437. [CrossRef] [PubMed]

35. Elsana, H.; Olusanya, T.O.B.; Carr-wilkinson, J.; Darby, S.; Faheem, A.; Elkordy, A.A. Evaluation of novel cationic gene based liposomes with cyclodextrin prepared by thin film hydration and microfluidic systems. Sci. Rep. 2019, 9, 15120. [CrossRef]

36. Ang, S.K.; Zhang, M.; Lodi, T.; Lu, H. Mitochondrial thiol oxidase Erv1: Both shuttle cysteine residues are required for its function with distinct roles. Biochem. J. 2014, 460, 199-210. [CrossRef]

37. Pinheiro, T.J.T.; Cheng, H.; Seeholzer, S.H.; Roder, H. Direct evidence for the cooperative unfolding of cytochrome $c$ in lipid membranes from H-2H exchange kinetics. J. Mol. Biol. 2000, 303, 617-626. [CrossRef]

38. Butterfield, D.A.; Sultana, R. Redox proteomics: Understanding oxidative stress in the progression of age-related neurodegenerative disorders. Expert Rev. Proteom. 2008, 5, 157-160. [CrossRef]

39. Turrens, J.F. Mitochondrial formation of reactive oxygen species. J. Physiol. 2003, 552, 335-344. [CrossRef]

40. Vogtle, F.N.; Burkhart, J.M.; Rao, S.; Gerbeth, C.; Hinrichs, J.; Martinou, J.C.; Chacinska, A.; Sickmann, A.; Zahedi, R.P.; Meisinger, C. Intermembrane space proteome of yeast mitochondria. Mol. Cell. Proteom. 2012, 11, 1840-1852. [CrossRef]

41. Di Fonzo, A.; Ronchi, D.; Lodi, T.; Fassone, E.; Tigano, M.; Lamperti, C.; Corti, S.; Bordoni, A.; Fortunato, F.; Nizzardo, M.; et al. The mitochondrial disulfide relay system protein GFER is mutated in autosomal-recessive myopathy with cataract and combined respiratory-chain deficiency. Am. J. Hum. Genet. 2009, 84, 594-604. [CrossRef]

42. Daithankar, V.N.; Schaefer, S.A.; Dong, M.; Bahnson, B.J.; Thorpe, C. Structure of the human sulfhydryl oxidase augmenter of liver regeneration and characterization of a human mutation causing an autosomal recessive myopathy. Biochemistry 2010, 49, 6737-6745. [CrossRef] [PubMed]

Publisher's Note: MDPI stays neutral with regard to jurisdictional claims in published maps and institutional affiliations.

(C) 2020 by the authors. Licensee MDPI, Basel, Switzerland. This article is an open access article distributed under the terms and conditions of the Creative Commons Attribution (CC BY) license (http://creativecommons.org/licenses/by/4.0/). 